Revue

Revue de l'histoire des religions

de Ihistoire des religions

Ulrike KRAMPL, Les secrets des faux sorciers. Police, magie et escroquerie à Paris au XVIII siècle

Paris, Éditions de l'EHESS («En temps et en lieux », 31), 2011

Mathilde Monge

\title{
OpenEdition
}

Journals

Édition électronique

URL : http://journals.openedition.org/rhr/8386

DOI : $10.4000 /$ rhr.8386

ISSN : 2105-2573

Éditeur

Armand Colin

Édition imprimée

Date de publication : 1 mars 2015

Pagination : 126-128

ISBN : 9782200929657

ISSN : 0035-1423

Référence électronique

Mathilde Monge, "Ulrike Krampl, Les secrets des faux sorciers. Police, magie et escroquerie à Paris au xvIII siècle », Revue de l'histoire des religions [En ligne], 1 | 2015, mis en ligne le 02 avril 2015, consulté le 22 septembre 2020. URL : http://journals.openedition.org/rhr/8386; DOI : https://doi.org/10.4000/rhr. 8386

Ce document a été généré automatiquement le 22 septembre 2020.

Tous droits réservés 


\section{Ulrike KRAMPL, Les secrets des faux sorciers. Police, magie et escroquerie à Paris au XVIII siècle}

Paris, Éditions de l'EHESS («En temps et en lieux », 31), 2011

Mathilde Monge

\section{RÉFÉRENCE}

Ulrike KRAMPL, Les secrets des faux sorciers. Police, magie et escroquerie à Paris au XVIII siècle, Paris, Éditions de l'EHESS (« En temps et en lieux », 31), 2011, 302 p., $23 €, 24 \mathrm{~cm}$, ISBN

978-2-7132-2322-8.

1 Le livre d'Ulrike Krampl, tiré de sa thèse de doctorat, s'attaque à un sujet classique, l'histoire de la sorcellerie, et à un lieu connu, Paris au XVIII siècle. Cependant, l'originalité consiste à traiter de la sorcellerie à Paris, au siècle des Lumières, sous un angle qui est plutôt celui de la police et de l'implémentation des normes, de l'anthropologie religieuse et de la sociologie. Le livre s'organise en trois temps : qu'estce qu'un «faux sorcier », quels sont les enjeux sociaux des secrets dont il est porteur, et enfin qu'est-ce que "croire » en la magie au XVIII siècle. Le religieux est présent de manière transversale dans tout l'ouvrage, car les sorciers constituent de plusieurs façons un objet et un sujet de concurrence : concurrence du public d'abord, qui va chercher dans une autre transcendance une solution pour faire face aux problèmes économiques d'ici-bas, de plus en plus prégnants, on le sait, à mesure que le siècle avance. Concurrence des autorités temporelles également (même si cela s'est joué dès le $\mathrm{XVI}^{\mathrm{e}}$ siècle) car au XVIII ${ }^{\mathrm{e}}$ siècle à Paris la sorcellerie relève de la police des mœurs. Les «faux sorciers" sont ici ceux qui proposent de lever des trésors, de conjurer des esprits, de transmuter le plomb en or.

2 L'A. emprunte à l'histoire de la police mais aussi à la sociologie de la déviance l'idée que le déviant, ici le sorcier, est « élaboré » par la police dans l'interaction avec le suspect et 
son entourage. Avec beaucoup de subtilité, et s'appuyant sur de nombreux exemples tirés des archives, elle montre que "c'est l'intervention [des policiers] qui fait apparaître - et non pas exister - la croyance ». Le processus de l'enquête, "saut d'obstacles », est retracé, de l'énonciation du soupçon à la mise à l'épreuve du secret, afin de démasquer la fausse sorcellerie, toujours dans le triptyque police-sorcier-public. Ce dernier a un rôle primordial: "instance d'accréditation", il permet aux actes magiques d'atteindre une certaine réalité. Il est aussi celui qui dénonce. Il est enfin celui qu'il faut protéger des «faux sorciers». Pour autant, l'A. n'est pas adepte d'une histoire décorporée : les nombreux exemples, la plupart du temps tirés des archives (voir le premier chapitre très détaillé) permettent également de saisir concrètement ce qu'étaient ces sorciers urbains du siècle des Lumières. Des transcriptions intégrales de documents d'archives en annexe (des inventaires de bibliothèques, un pacte avec le diable et une lettre) enrichissent ces portraits.

3 La deuxième partie s'articule autour du "secret de magie ", terme usité dans les archives, et de ses usages. Il apparait central au XvIII siècle, et l'A. le traite comme un "fait social total», en articulation avec un autre terme d'archive, préoccupation constante de la police naissante, «le public». Dans cette partie, il s'agit en fait de comprendre pourquoi les policiers du Paris des Lumières s'intéressaient tant à des personnages plutôt « désuets ». Ce « secret » traverse les différentes couches sociales : l'affaire des poisons n'est ainsi qu'un des nombreux cas où la plus haute noblesse s'est adjoint les services d'une personne du peuple. Par ailleurs, le problème du secret pour les autorités, et ce qui le fait exister, c'est précisément sa publicité : la marque du secret est un «signe de distinction qui permet ainsi la constitution d'espaces ou de groupes exclusifs au sein desquels la transmission de savoirs et de savoir-faire réservés [...] se trouve seule autorisée ». Les secrets se disséminent dans la ville et créent cet espace social relationnel par le biais de la séduction: autrefois attribut du Malin, elle est devenue au XVIII ${ }^{\mathrm{e}}$ siècle le propre des faux sorciers. Ils créent ainsi un espace autre, et la dimension politique du problème des faux sorciers ne réside pas dans la «magie » ellemême, à laquelle les lieutenants-généraux de police ne croient pas, mais dans l'art de «forger des liens horizontaux " susceptibles de cultiver la désaffection à l'égard de l'autorité du souverain. Enfin, les secrets des faux sorciers ressortissent au registre économique, car il s'agit bien de retrouver ou de créer de la richesse (la plupart du temps de l'or), moyennant finance. C'est par ailleurs au XviII siècle que prend forme la catégorie juridique de l'escroc, qui auparavant se confondait avec celle des voleurs. La catégorie devient autonome car l'appréhension de l'imagination se modifie. Autrefois espace de construction et de représentation de ce qui n'est pas, porte ouverte à l'influence du diable, on passe à un espace de représentation des illusions et des chimères d'où le diable est absent. Les faux sorciers incitent les "crédules " à abandonner toute volonté. L'évolution que connaît le xvIII ${ }^{\mathrm{e}}$ siècle est le passage du délit de sorcellerie du domaine du politique à celui du délit économique, l'escroquerie.

L'A. se penche enfin sur les dynamiques du secret, dans une dernière partie un peu hybride. Il s'agit tout d'abord de situer l'habitat et l'activité des faux sorciers, entreprise précieuse et assortie de plusieurs cartes, à l'échelle urbaine et régionale (une vaste Île-de-France). Elle montre ainsi que la résidence des faux sorciers les met « au cœur» de leur public, habitant dans les quartiers les plus peuplés, mais pas nécessairement les plus pauvres ou ceux qui sont à l'écart dans la ville. La ville «magique », les lieux où les secrets de magie se « réalisent » ou se transmettent, est par 
contre aussi bien au centre qu'en périphérie ou hors de la ville. Le temps de la magie est lui aussi étroitement imbriqué avec le temps ordinaire, et avec le temps de l'Église: l'invocation des esprits est impossible autour du temps pascal, le temps de la magie respecte donc le calendrier liturgique. Cependant nombre de rituels magiques sont mesurés ou réalisés dans le temps sacré : «la femme Guillaume conseille "messes, voyages [vers Notre-Dame-des-Vertus] et poudres" pour faire aboutir mariages et successions » (p. 212). La magie est donc imbriquée avec le religieux, sans surprise, mais il s'agit aussi d'une considération pratique. L'« espace d'un Credo à dire» (p. 206), nécessaire pour fabriquer un sirop, est aussi un moyen de mesurer le temps sans horloge, et on trouve aussi bien, dans un traité à passer avec le diable, une mesure du temps plus "moderne", en minutes, dont l'usage s'impose progressivement au XVIII siècle. Le livre s'achève par une analyse des "figures du croire ", à travers l'étude des conditions d'énonciation de la croyance dans le cadre des interrogatoires : il s'agit de l'étudier à travers «une parole sur soi qui tient nécessairement compte de l'enchevêtrement de situation d'énonciation aux règles opposées ». Il faut par exemple dénier la part magique des "secrets » délivrés, et expliquer qu'une poudre donnée à une jeune fille sert à blanchir les dents. Les accusés sont souvent pris entre une religion chrétienne à laquelle ils adhèrent, par laquelle ils doivent prêter serment, et des pratiques qu'ils savent être répréhensibles au regard des normes. Certains, " convaincus d'être dans la bonne religion, finissent par s'en faire une représentation qui leur est propre.» (p. 229). Ainsi l'A. montre très bien que la magie, c'est-à-dire la croyance, n'est pas un horizon unique : la distinction avec la foi n'est pas toujours nette et elle " ne se substitue pas au travail, ni aux affects [...] elle ne fait que s'y mêler, de temps à autre » (p. 233).

En conclusion, l'A. propose une interprétation diachronique globale, malgré quelques indications ponctuelles au cours du livre. Le phénomène des "faux sorciers", caractéristique des villes de la première moitié du XVIII siècle, participe à la "redéfinition de la frontière entre imagination et réalité ». Dans la deuxième moitié du siècle, la fausseté étant établie, la catégorie judiciaire disparaît (le sorcier est forcément faux). Les secrets, eux, subsistent. Avec un travail d'archives remarquable, le livre d'Ulrike Krampl apporte une contribution importante à l'histoire urbaine comme à l'histoire du rapport à la croyance, au moment où nombreux sont ceux qui appellent à faire de la raison le critère de toute chose. Elle montre bien que dans ce domaine la distinction entre culture populaire et culture des élites n'est pas opérante, la ligne de partage qu'est la crédulité étant beaucoup plus floue et devant être à chaque fois située.

\section{AUTEURS}

\section{MATHILDE MONGE}

Université Toulouse 2 - Jean Jaurès. 